\title{
ON THE OVERCONVERGENCE OF SEQUENCES OF POLYNOMIALS OF BEST APPROXIMATION*
}

\author{
BY
}

J. L. WALSH

1. Introduction. There are many cases where the sequence of polynomials of best approximation to an analytic function in a given region $C$ converges to that function (or its analytic extension) not merely in $C$ but in a larger region containing $C$ in its interior. It is the object of the present paper to show that many such results can be derived easily from a single general theorem on the degree of approximation to an analytic function. We shall discuss also the corresponding facts for approximation to harmonic functions by harmonic polynomials; the analogy between the two theories is close. There are a number of cases to be treated, according as best approximation is measured (1) in the sense of Tchebycheff, (2) by line integrals taken over the curve bounding the region considered, (3) by line integrals taken over the unit circle in a new plane, after conformal mapping of the original region onto the interior of this circle, or (4) by surface integrals over the original region.

The term overconvergence is used to denote the behavior mentioned above, that although a given function is defined and approximated in a certain region, the sequence of polynomials approximating the function in the given region may converge uniformly in a larger region containing the given region in its interior. The term overconvergence has recently been used by Ostrowski in a somewhat different connection. Our primary topic is the overconvergence of sequences of polynomials, but we devote some space $(\$ \$ 11,12)$ also to approximation where overconvergence need not take place.

Theorem I. Let $C$ be an arbitrary closed limited point set (not a single point) of the z-plane, whose complementary set with respect to the entire plane is simply connected. Let $w=\phi(z)$ be a function which maps conformally the exterior of $C$ onto the exterior of the unit circle in the w-plane so that the points at infinity correspond to each other. Let $C_{R}$ denote the curve $|\phi(z)|=R>1$, that is, the transform in the $z$-plane of the circle $|w|=R$.

* Presented to the Society, February 22, 1930; received by the editors April 7, 1930. An abstract of this paper was published in the Proceedings of the National Academy of Sciences, vol. 16 (1930), p. 297. 
If the function $f(z)$ is analytic on and within $C_{R}$, then there exist polynomials $p_{n}(z)$ of respective degrees $n, n=0,1,2, \cdots$, such that the inequalities

$$
\left|f(z)-p_{n}(z)\right| \leqq \frac{M}{R^{n}}, \quad R>1,
$$

where $M$ is independent of $n$ and of $z$, are valid for every $z$ in $C$.

If the polynomials $p_{n}(z)$ are given so that (1) is valid for every $z$ in $C$, then the sequence $\left\{p_{n}(z)\right\}$ converges everywhere interior to $C_{R}$ and uniformly on any closed point set interior to $C_{R}$, and thus $f(z)$ is analytic throughout the interior of $C_{R}$ * $^{*}$

2. Tchebycheff polynomials. The Tchebycheff polynomial of degree $n$ for approximation to $f(z)$ on $C$ is the polynomial $\pi_{n}(z)$ of degree $n$ such that

$$
\max \left|f(z)-\pi_{n}(z)\right|, \quad z \text { on } C,
$$

is less than

$$
\max \left|f(z)-p_{n}(z)\right|, \quad z \text { on } \mathfrak{C},
$$

for any other polynomial $p_{n}(z)$ of degree $n$. The Tchebycheff polynomial exists and is unique. $\dagger$ The present writer has already proved elsewheref the following theorem, but the proof is so short and so typical of the reasoning used later that it will be given here. The theorem was first proved by Faber§, using an entirely different method, and only in the restricted case that $C$ is bounded by an analytic Jordan curve.

Theorem II. Let $C$ and $C_{R}$ have the same significance as in Theorem I. If the function $f(z)$ is analytic interior to $C_{R}$, then the sequence $\left\{\pi_{n}(z)\right\}$ of Tchebycheff polynomials for approximation to $f(z)$ on $C$ converges to $f(z)$ interior to $C_{R}$, uniformly on an arbitrary closed point set interior to $C_{R}$.

By the first part of Theorem I, if $R_{1}$ be an arbitrary number less than $R$, there exist polynomials $p_{n}(z)$ of respective degrees $n$ such that

$$
\left|f(z)-p_{n}(z)\right| \leqq \frac{M}{R_{1}^{n}}, \quad z \text { on } C .
$$

* Various parts of Theorem I are due to various writers, notably S. Bernstein, M. Riesz, Faber, Fjeér, Szegö. See Walsh, Münchner Berichte, 1926, pp. 223-229. An arbitrary function of the form $a_{0}+a_{1} z+\cdots+a_{n} z^{n}$ is said to be a polynomial of degree $n$.

† Tonelli, Annali di Matematica, (3), vol. 15 (1908), pp. 47-119.

$\ddagger$ See Bulletin of the American Mathematical Society, vol. 35 (1929), pp. 499-544, especially pp. 513, 514, and also Boundary values of an analytic function and the Tchebycheff method of approximation, these Transactions vol. 32 (1930), pp. 335-390.

\& Crelle's Journal, vol. 150 (1920), pp. 79-106; especially p. 105. 
This inequality, holding for the polynomials $p_{n}(z)$, must also hold for the Tchebycheff polynomials:

$$
\left|f(z)-\pi_{n}(z)\right| \leqq \frac{M}{R_{1}{ }^{n}}, \quad z \text { on } C,
$$

and since $R_{1}<R$ is arbitrary, Theorem II follows from the second part of Theorem I.

If $C^{\prime}$ is an arbitrary closed limited region, we denote by $C$ the totality of closed limited regions into which the boundary of $C^{\prime}$ divides the plane; the boundary points of $C$ (considered as a set of regions, not as a point set) are precisely the boundary points of $C^{\prime}$, and if inequality (1) holds for all points of $C^{\prime}$, or even for all points of the boundary of $C^{\prime}$, then (1) holds also for all points of $C$. The curve $C_{R}$ is defined as in Theorem $I$ from this point set $C$, by means of the mapping of the complement of $C$. Thus, if the function $f(z)$ is analytic interior to $C_{R}$, then the sequence $\left\{\pi_{n}(z)\right\}$ of Tchebycheff polynomials for approximation to $f(z)$ on $C^{\prime}$ converges to $f(z)$ interior to $C_{R}$, uniformly on an arbitrary closed point set interior to $C_{R}$. It will be noticed, in fact, that the Tchebycheff polynomial is the same for approximation to $f(z)$ on $C$, or on $C^{\prime}$, or on the boundary of $C$ or $C^{\prime}$. The function in the left-hand member of (1) has one and the same maximum for all these point sets.

Throughout the present paper we shall use the notation $C_{R}$ in the following sense. Let $C$ be an arbitrary closed limited point set and $D$ the set of points which can be joined to the point at infinity by Jordan arcs which do not meet $C$. Then the image of $|w|=R>1$ when $D$ is mapped onto the exterior of $|w|=1$ in the $w$-plane so that the points at infinity correspond to each other shall be denoted by $C_{R}$.

3. Tchebycheff approximation with a norm function. The notion of approximation by the method of Tchebycheff with a norm function will now be introduced. Let the function $p(z)$ be given continuous and different from zero on $C$. Then a Tchebycheff polynomial for approximation to $f(z)$ on $C$ with the norm function $p(z)$ is a polynomial $\pi_{n}^{\prime}(z)$ of degree $n$ such that

$$
\max \left[|p(z)|\left|f(z)-\pi_{n}^{\prime}(z)\right|\right], \quad z \text { on } C
$$

is no greater than

$$
\max \left[|p(z)|\left|f(z)-p_{n}^{\prime}(z)\right|\right], \quad z \text { on } C,
$$

for any other polynomial $p_{n}^{\prime}(z)$ of degree $n$. The existence of the polynomial 
$\pi_{n}^{\prime}(z)$ can easily be proved* and the uniqueness follows from the original method of Tonelli. $†$ If $C$ and $C_{R}$ have the same significance as in Theorem I and if $f(z)$ is analytic within $C_{R}$, then the sequence of Tchebycheff polynomials $\left\{\pi_{n}^{\prime}(z)\right\}$ for approximation to $f(z)$ on $C$ with an arbitrary norm function $p(z)$ converges to $f(z)$ interior to $C_{R}$, uniformly on an arbitrary closed point set interior to $C_{R}$.

If $R_{1}$ is an arbitrary number less than $R$, the first part of Theorem I informs us that a sequence of polynomials $p_{n}(z)$ of respective degrees $n$ exists so that

$$
\left|f(z)-p_{n}(z)\right| \leqq \frac{M}{R_{1}^{n}}, \quad z \text { on } C .
$$

If we have $|p(z)| \leqq P$ for $z$ on $C$, we have also

$$
|p(z)|\left|f(z)-p_{n}(z)\right| \leqq \frac{M P}{R_{1}^{n}}, \quad z \text { on } C,
$$

from which follows

$$
|p(z)|\left|f(z)-\pi_{n}^{\prime}(z)\right| \leqq \frac{M P}{R_{1}{ }^{n}}, \quad z \text { on } C .
$$

If we have $|p(z)| \geqq q>0, z$ on $C$, it follows that

$$
\left|f(z)-\pi_{n}{ }^{\prime}(z)\right| \leqq \frac{M P}{q R_{1}{ }^{n}}, \quad z \text { on } C,
$$

and our result is an immediate consequence of the second part of Theorem I.

If we consider the ordinary Tchebycheff polynomial $\pi_{n}(z)$, it is of course a matter of indifference whether we consider approximation to $f(z)$ on $C$, or approximation to $f(z)$ on the boundary $\Gamma$ of $C$, for the function in the left-

* The proof as given in the case of reals for the ordinary Tchebycheff polynomial applies without essential change. See for instance de la Vallée Poussin, A pproximation des Fonctions, Paris, 1919, p. 74.

$\dagger$ Loc. cit. The principal modifications to be made in his treatment are the following (in his notation, p. 109, except for the norm function $n(x))$ :

Choose $\epsilon<\mu / 2$ and $\delta$ so that $\left|x^{\prime}-x^{\prime \prime}\right|<\delta$ implies

$$
\mu=\max |Y(x) n(x)|, P_{n}\left(x_{r}\right)=Y\left(x_{r}\right) .
$$

$$
\left|Y\left(x^{\prime}\right) n\left(x^{\prime}\right)-Y\left(x^{\prime \prime}\right) n\left(x^{\prime \prime}\right)\right|<\epsilon,\left|P\left(x^{\prime}\right) n\left(x^{\prime}\right)-P\left(x^{\prime \prime}\right) n\left(x^{\prime \prime}\right)\right|<\epsilon .
$$

Choose $\omega$ so that, in the circles $\left|x_{r}-x\right| \leqq \delta$, we have

and so that on the entire point set $A$ we have

$$
\left|Y(x)-\omega P_{n}(x)\right||n(x)|<\mu
$$

$$
\omega\left|P_{n}(x)\right||n(x)|<\frac{\mu-\mu^{\prime \prime}}{2},
$$

where in $A^{\prime}$

$$
|Y(x) n(x)| \leqq \mu^{\prime \prime}<\mu .
$$


hand member of (1) has its maximum in $C$ on $\Gamma$. A similar remark holds for the polynomial $\pi_{n}^{\prime}(z)$, if the norm function $p(z)$ is analytic on $C$.

4. Approximation in the sense of least $p$ th powers. We turn now to consideration of polynomials of best approximation in the sense of least $p$ th powers. Let $p$ be an arbitrary positive number greater than unity, ${ }^{*}$ and let the point set $C$ now be the closed interior of a rectifiable Jordan curve $\Gamma$. Let $n(s)$ be an arbitrary function continuous and positive on $\Gamma$, where $s$ indicates arc length on $\Gamma$; this weight or norm function is usually chosen as unity, but that is not necessary. A polynomial of best approximation is a polynomial $\pi_{n}(z)$ of degree $n$ such that

$$
\int_{\Gamma}\left|f(z)-\pi_{n}(z)\right|{ }^{p} n(s) d s
$$

is not greater than the corresponding expression

$$
\int_{\Gamma}\left|f(z)-p_{n}(z)\right| p_{n}(s) d s
$$

formed for any other polynomial $p_{n}(z)$ of degree $n$. The polynomial $\pi_{n}(z)$ can easily be shown to exist; its uniqueness follows from the method recently used by Julia $\dagger$ in a similar situation.

TheOREM III. Let the function $f(z)$ be analytic within $C_{R}$, where the point set $C$ is the interior of the rectifiable Jordan curve $\Gamma$. Then the sequence of polynomials $\left\{\pi_{n}(z)\right\}$ of best approximation to $f(z)$ on $C$ in the sense of least weighted pth powers as measured on $\Gamma$ converges to $f(z)$ throughout the interior of $C_{R}$, uniformly on any closed point set interior to $C_{R}$.

We shall have occasion to use the following well known inequality for arbitrary continuous functions $F(x)$ and $G(x)$ :

$$
\left|\int[F(x)]^{\alpha}[G(x)]^{1-\alpha} d x\right| \leqq\left(\int|F(x)| d x\right)^{\alpha}\left(\int|G(x)| d x\right)^{1-\alpha} .
$$

If $R_{1}$ is an arbitrary number less than $R$, there exist by Theorem I polynomials $p_{n}(z)$ of respective degrees $n$ such that

$$
\left|f(z)-p_{n}(z)\right| \leqq \frac{M}{R_{1}^{n}}, \quad z \text { on } \Gamma .
$$

From this it follows that $M^{\prime}$ exists such that

* Much of our work is valid if $p$ is assumed merely positive.

$\dagger$ Acta Litterarum ac Scientiarum (Szeged), vol. 14 (1929), pp. 217-226. 


$$
\int_{\Gamma}\left|f(z)-p_{n}(z)\right|^{p} n(s) d s \leqq \frac{M^{\prime}}{R_{1}^{n p}}
$$

whence we deduce the corresponding inequality for the polynomials $\pi_{n}(z)$ of best approximation:

$$
\int_{\Gamma}\left|f(z)-\pi_{n}(z)\right|^{p} n(s) d s \leqq \frac{M^{\prime}}{R_{1}^{n p}} .
$$

The function $n(s)$, being positive and continuous on $\Gamma$, has a positive minimum $n^{\prime}$ on $\Gamma$, so we may write

$$
\int_{\Gamma}\left|f(z)-\pi_{n}(z)\right|^{p} d s \leqq \int_{\Gamma}\left|f(z)-\pi_{n}(z)\right|^{p} \frac{n(s)}{n^{\prime}} d s \leqq \frac{M^{\prime}}{n^{\prime} R_{1}^{n p}}=\frac{M_{1}^{\prime}}{R_{1}^{n p}} .
$$

Here we apply Cauchy's integral formula

$$
f(z)-\pi_{n}(z)=\frac{1}{2 \pi i} \int_{\Gamma}^{-} \frac{f(t)-\pi_{n}(t)}{t-z} d t,
$$

and use (4) in conjunction with (2), setting $\alpha=1 / p, F(x)=\left|f(t)-\pi_{n}(t)\right|^{p}$, $G(x)=1 /|t-z|^{p /(p-1)}, d x=|d t|$. If we so restrict $z$ as to lie on an arbitrary closed point set interior to $\Gamma$, we have then

$$
\left|f(z)-\pi_{n}(z)\right| \leqq \frac{M^{\prime \prime \prime}}{R_{1}^{n}}
$$

where $M^{\prime \prime \prime}$ depends on the particular point set to which $z$ is restricted.

For definiteness we consider (5) to hold on an arbitrary Jordan curve $\Gamma^{\prime}$ interior to $\Gamma$. The sequence $\left\{\pi_{n}(z)\right\}$ then converges, by the second part of Theorem I, interior to the curve $C_{R_{1}}$, and uniformly on any closed point set interior to $C_{R_{1}^{\prime}}^{\prime}$, where $C_{R_{1}^{\prime}}$ is the image in the $z$-plane of the circle $|w|=R_{1}$ when the exterior of $\Gamma^{\prime}$ is mapped onto the exterior of the unit circle in the $w$-plane so that the points at infinity correspond to each other. When $\Gamma^{\prime}$ approaches $\Gamma$ uniformly, the curve $C_{R_{1}^{\prime}}$ approaches $C_{R_{1}}$ uniformly. ${ }^{*}$ Since $R_{1}$ (less than $R$ ) is arbitrary, and since the convergence of the sequence $\left\{\pi_{n}(z)\right\}$ is uniform on any closed point set interior to $C_{R_{1}^{\prime}}$, Theorem III is established.

Theorem III was proved for the case $p=2, n(s) \equiv 1$ by Szegö $\dagger$ for the case that $\Gamma$ is an analytic curve and by Smirnoff $\ddagger$ for the case that $\Gamma$ is the most

* Carathéodory, Mathematische Annalen, vol. 72 (1912), pp. 107-144, especially pp. 126, 127.

$\dagger$ Mathematische Zeitschrift, vol. 9 (1921), pp. 218-270.

$\ddagger$ Journal de la Société Physico-Mathématique de Léningrade, vol. 2 (1928), pp. 155-178, especially pp. 177, 178 . 
general rectifiable curve. Theorem III was also proved by Szegö for the case $p=2$ and $\Gamma$ a circle. ${ }^{*}$ In all these cases the method was different from the present one. Indeed, under the present hypothesis, the polynomial of best approximation to an arbitrary function $f(z)$ is of the form

$$
\pi_{n}(z)=a_{0} P_{0}(z)+a_{1} P_{1}(z)+\cdots+a_{n} P_{n}(z),
$$

where the polynomials $P_{i}(z)$ depend on $\Gamma$ but not on $f(z)$, and where the coefficients $a_{i}(i \leqq n)$ are independent of $n$. The method used by both Szegö and Smirnoff is the study of the asymptotic properties of the polynomials $P_{i}(z)$ and of the coefficients $a_{i}$. The first part of Theorem I is used by both writers.

A less specific result than Theorem III for certain more restricted types of curves has recently been established by Dunham Jackson. $\dagger$

We have proved actually more than is stated in Theorem III, for we have used in the proof only inequality (3), by way of hypothesis on the polynomials $\pi_{n}(z)$.

5. Least $p$ th powers and conformal mapping; Jordan regions. There is a result analogous to Theorem III which applies even if the curve $\Gamma$ is not rectifiable. This consists in measuring approximation not on $\Gamma$ but on $\gamma$, the unit circle in the $w$-plane, after mapping the interior of $\Gamma$ onto the interior of $\gamma$, setting $w=\phi(z), z=\psi(w)$. This method has recently been used with success by Julia, $\ddagger$ in the analogous situation of approximation of harmonic functions by harmonic polynomials. Indeed, the present paper would probably not have been written without the impetus due to his paper.

Let the function $n(\theta)$ be positive and continuous on $\gamma$, where $\theta$ is arc length on $\gamma$; we consider as the polynomial of degree $n$ of best approximation the polynomial $\pi_{n}(z)$ of degree $n$ such that

$$
\int_{\gamma}\left|f(z)-\pi_{n}(z)\right|^{p} n(\theta) d \theta, \quad z=\psi(w),
$$

is less than the corresponding expression for any other polynomial of degree $n$, where $f(z)$ is given analytic on and within $\Gamma$. The notation $\gamma$ is to be used later as well; by measure of approximation with least $p$ th powers on $\gamma$ we shall understand the integral (6) or (for harmonic functions) the integral analogous to it; the given region $C$ is to be mapped conformally on the

* Mathematische Zeitschrift, vol. 6 (1920), pp. 167-120.

† On certain problems of approximation in the complex domain, presented to this Society December 28, 1927, abstract published in the Bulletin of the American Mathematical Society, vol. 34 (1928,) p. 151 ; the paper is expected to appear in the same Bulletin. The abstract does not state the principal results of the paper, and that paper and the present one were written entirely independently of each other. The methods used are different in the two cases.

$\ddagger$ Loc. cit. An arbitrary point interior to $\Gamma$ is made to correspond to the center of $\gamma$. 
interior of $\gamma$. Theorem IVa will be proved by almost precisely the method used in the proof of Theorem III. As in Theorem III, the polynomial $\pi_{n}(z)$ exists and is unique; this may be proved by the method of Julia.

THeOREM IVa. Let the function $f(z)$ be analytic within $C_{R}$, where $C$ is the interior of the Jordan curve $\Gamma$. Then the sequence of polynomials $\left\{\pi_{n}(z)\right\}$ of best approximation to $f(z)$ on $C$ in the sense of least weighted pth powers as measured on $\gamma$ converges to $f(z)$ throughout the interior of $C_{R}$, uniformly on any closed point set interior to $C_{R}$.

We can proceed as in the proof of Theorem III, now (as in expression (6) ) making frequent use of the theorem that the mapping of the interior of $\Gamma$ onto the interior of $\gamma$ is continuous in the closed regions. Inequality (3) holds, where the integral is now taken over $\gamma$ instead of $\Gamma$; the values of the integrand on $\gamma$ are the values of the functions at the corresponding points of $\Gamma$. Cauchy's integral formula is likewise valid, the integral being taken over $\gamma$, and hence (5) holds for $w$ on any closed point set interior to $\gamma$, that is, for $z$ on any closed point set interior to $\Gamma$; the number $M^{\prime \prime \prime}$ depends naturally on this point set. This point set can be chosen the closed interior of a Jordan curve $\Gamma^{\prime}$ interior to $\Gamma$, so by the reasoning previously given based on the results of Carathéodory, Theorem IVa now follows.

The case of Theorem IVa for $p=2, n(\theta) \equiv 1$, is of particular interest, for in this case approximation is in the sense of least squares on $\gamma$, and the polynomial $\pi_{n}(z)$ is of the form*

$$
\pi_{n}(z)=a_{0} P_{0}(z)+a_{1} P_{1}(z)+\cdots+a_{n} P_{n}(z),
$$

where the polynomials $P_{i}(z)$ depend on the region $C$ and not on the function $f(z)$ and where the coefficients $a_{i}(i \leqq n)$ are independent of $n$. This yields, then, a sequence of polynomials $\left\{P_{n}(z)\right\}$ in terms of which can be developed an arbitrary function $f(z)$ analytic on and within $\Gamma$, and the development

$$
a_{0} P_{0}(z)+a_{1} P_{1}(z)+\cdots+a_{n} P_{n}(z)+\cdots
$$

converges uniformly to the function $f(z)$ on and within $\Gamma$. This problem for the most general Jordan curve $\Gamma$ was long ago proposed by Faber†; a solution was more recently announced by Fejér, $\ddagger$ but so far as the present writer is aware the details have not yet been published.

These polynomials $\left\{P_{n}(z)\right\}$ may be obtained by starting with the special polynomials $\left\{z^{n}\right\}$, and orthogonalizing and normalizing this latter set with

* Compare Szegö, loc. cit.

$\dagger$ Mathematische Annalen, vol. 57 (1903), pp. 389-408.

† Göttii:ger Nachrichten, 1918, pp. 319-331. 
respect to integration on $\gamma$. A corresponding remark holds naturally in every case below, for $p=2, n(s) \equiv 1$.

Theorem IVa is closely related to Theorem III, for there is a relation between arc length on $\Gamma$ (if $\Gamma$ is rectifiable) and arc length on $\gamma$, and the functions $n(s)$ and $n(\theta)$ are arbitrary. The writer knows, however, no proof that Theorem III is contained in Theorem IVa.

6 . Least $p$ th powers and conformal mapping; general regions. Theorem IVa is in reality but a special case, proved first because of its simplicity, of a more general theorem:

Theorem IV. Let $C$ be an arbitrary closed limited simply connected region and let the function $f(z)$ be analytic interior to $C_{R}$. Then the sequence of polynomials $\left\{\pi_{n}(z)\right\}$ of best approximation to $f(z)$ on $C$ in the sense of least weighted pth powers as measured on $\gamma$ converges to $f(z)$ throughout the interior of $C_{R}$, uniformly on any closed point set interior to $C_{R}$.

Under the conformal map $w=\phi(z), z=\psi(w)$ of $C$ onto the interior of $\gamma$, almost all (i.e. all, except possibly for points of a set of measure zero) points of $\gamma$ correspond to accessible boundary points of $C$. For, as Carathéodory has pointed out, the function $\psi(w)$ is limited interior to $\gamma$, and hence by Fatou's theorem $\lim _{r \rightarrow 1, r<1} \psi\left(r e^{i \theta}\right)$ exists for almost all values of $\theta$. A radius $0 \leqq r \leqq 1, \theta$ constant, for which this limit exists therefore corresponds under the conformal map to a Jordan arc from the point $\psi(0)$ to a boundary point of $C$. When this limit exists for a particular value of $\theta$, we define $\psi\left(e^{i \theta}\right)$ as this limit.

The functions $f(z), p_{n}(z)$ (about to be introduced), $\pi_{n}(z)$ are to be defined on $\gamma$ after conformal transformation by means of their limiting values by radial approach to $\gamma$; these limiting values exist for almost all values of $\theta$, by Fatou's theorem. Moreover, for any point of $\gamma$ corresponding to an accessible point $z^{\prime}$ of the boundary of $C$, and thus for almost all points of $\gamma$, these limiting values exist and coincide with the values of the functions $f(z)$, $p_{n}(z), \pi_{n}(z)$ at the point $z^{\prime}$. For radial approach to $w=\phi\left(z^{\prime}\right)$ corresponds to approach to $z^{\prime}$ along a Jordan arc, and for such approach the functions involved have limiting values, $f\left(z^{\prime}\right), p_{n}\left(z^{\prime}\right), \pi_{n}\left(z^{\prime}\right)$.

Cauchy's integral formula applies in the w-plane to any function such as $f(z), p_{n}(z), \pi_{n}(z)$, the integral now being taken in the sense of Lebesgue. We have, for instance,

$$
f[\psi(w)]=\frac{1}{2 \pi i} \int_{\gamma^{\prime}} \frac{f[\psi(t)]}{t-w} d t, \quad t=\rho e^{i \theta},
$$

where $\gamma^{\prime}$ is the circle $|w|=\rho<1$. Keep $w$ fixed. When $\rho$ approaches unity 
through a sequence of values, the integrand, considered as a function of $\theta$, approaches the limit $f[\psi(t)] /(t-w)$ for almost all values of $\theta$. During this approach of $\rho$ to the limit unity the integrand remains uniformly limited. It follows that Cauchy's formula

$$
f[\psi(w)]=\frac{1}{2 \pi i} \int_{\gamma} \frac{f[\psi(t)]}{t-w} d t
$$

is valid; a similar result naturally holds for the functions $p_{n}(z), \pi_{n}(z)$.

By Theorem I there exist polynomials $p_{n}(z)$ of respective degrees $n$ such that

$$
\left|f(z)-p_{n}(z)\right| \leqq \frac{M}{R_{1}^{n}}, \quad z \text { on } C .
$$

This same inequality is valid almost everywhere for $w$ on $\gamma$, for the two methods of defining the functions $f(z)$ and $p_{n}(z)$ on $\gamma$, namely by radial approach and by direct correspondence $w=\phi(z), z=\psi(w)$, are equivalent almost everywhere on $\gamma$. Hence we have

$$
\int_{\gamma}\left|f(z)-p_{n}(z)\right|^{p} n(\theta) d \theta \leqq \frac{M^{\prime}}{R_{1}^{n_{p}}},
$$

from which follows inequality (5) as before.

The proof of the theorem now follows without essential change from the proof given for Theorem IVa; the relation of the curves $C_{R_{1}^{\prime}}$ and $C_{R_{1}}$ is discussed in more detail below in connection with Theorem V.

7. Generalizations. Theorems III and IV persist even for the case $p=1$. The proofs in that case are even more simple than the proofs we have given in detail, and the modifications are left to the reader. This is true for all of the later theorems to be proved; they are valid also for $p=1$, although the proofs are slightly different from those given for an arbitrary value of $p$ greater than unity.

There are cases of approximation analogous to Theorems II, III, and IV, where approximation with an arbitrarily small preassigned error is not possible. For instance, the function $f(z)$ may no longer be analytic interior to $C$ but may have a singularity there. Or the function $f(z)$ may be analytic as prescribed in Theorem III, but the approximating functions may (in Theorems II, III, or IV) all be required to take on values at prescribed points interior to $C$ different from the values of $f(z)$ at those points. In the simplest cases, this subject has been treated by F. Riesz, ${ }^{*}$ Julia, $\dagger$ and the

* Acta Mathematica, vol. 42 (1919), pp. 145-171.

† Bulletin des Sciences Mathématiques, vol. 62 (1927); pp. 370-384; Annales de l'École Normale, (3), vol. 44 (1927), pp. 289-309. 
present writer.* The complete extensions of Theorems III and IV still remain to be given, however, and the present writer hopes to consider them on another occasion. We remark, though, that the requiring (in addition to the other requirements) of the approximating functions to coincide with the functions approximated at a finite number of points interior to $C$ does not alter any of the results of the present paper.

8. Surface integrals. Thus far we have considered primarily line integrals as measures of the closeness of approximation. We now take up the possibility of using surface integrals instead. Let $C$ be an arbitrary closed Jordan region, let the function $f(z)$ be analytic in the closed region, and let the norm function $n(z)$ be positive and continuous in $C$. The measure of approximation of the polynomial $p_{n}(z)$ to $f(z)$ shall be $\dagger$

$$
\iint_{C}\left|f(z)-p_{n}(z)\right|^{p} n(z) d S,
$$

and it follows from the method used by Julia (loc. cit.) that the polynomial $\pi_{n}(z)$ of best approximation exists and is unique. We now prove a result for more general regions than Jordan regions.

THEOREM V. Let $C$ be an arbitrary limited closed region. If the function $f(z)$ is analytic interior to $C_{R}$, then the sequence $\left\{\pi_{n}(z)\right\}$ of polynomials of best approximation to $f(z)$ in the sense of least weighted pth powers over the area $C$ converges to $f(z)$ interior to $C_{R}$, uniformly on an arbitrary closed point set interior to $C_{R}$.

It is of course true (compare $\$ 2$ ) that the boundary of $C$ may separate the plane into more than two regions. Let $D$ denote the region which is the aggregate of all points which can be connected with the point at infinity by Jordan arcs which do not meet $C$. The curve $C_{R}$ is defined as usual as the transform of the circle $|w|=R$ when $D$ is mapped onto the exterior of $|w|=1$ in such a way that the points at infinity in the $z$ - and w-planes correspond to each other. The region $C$ of course lies interior to $C_{R}$. Theorem I applies here, if the point set $C$ (of Theorem $\mathrm{I}$ ) is interpreted as the complement (with the respect to the entire plane) of $D$.

Let $R_{1}$ be an arbitrary positive number less than $R$. Then by Theorem I there exist polynomials $p_{n}(z)$ of respective degrees $n$ such that

$$
\left|f(z)-p_{n}(z)\right| \leqq \frac{M}{R_{1}{ }^{n}}, \quad z \text { on } C .
$$

\footnotetext{
* See the reference in the footnote in connection with Theorem II.

$\dagger$ In case the boundary of $C$ has area, either upper or lower integral may be used here.
} 
Thus we have (7) less than or equal to some $M^{\prime} / R_{1}{ }^{n p}$; the same inequality holds if $p_{n}(z)$ is replaced by $\pi_{n}(z)$, and when we notice that $n(z)$ has a positive minimum on $C$, we have

$$
\iint_{C}\left|f(z)-\pi_{n}(z)\right|^{p} d S \leqq \frac{M^{\prime \prime}}{R_{1}^{n p}} .
$$

Let $K$ be an arbitrary circle of radius $r$ interior to $C$. Then we have

$$
f(z)-\pi_{n}(z)=\frac{1}{\pi r^{2}} \iint_{K}\left[f(t)-\pi_{n}(t)\right] d S,
$$

where $z$ denotes the center of $K$ and $t$ is the variable of integration. In fact this relation can be deduced by a single integration from Gauss's meanvalue theorem for harmonic functions.* Let us apply inequality (2), setting $F(x)=\left|f(t)-\pi_{n}(t)\right|^{p}, \alpha=1 / p, G(x)=1$. We have

$$
\left|f(z)-\pi_{n}(z)\right| \leqq \frac{1}{\left(\pi r^{2}\right)^{1 / p}}\left\{\iint_{K}\left|f(t)-\pi_{n}(t)\right|^{p} d S\right\}^{1 / p}:
$$

the integral over $K$ is not greater than the integral over $C$, so we have

$$
\left|f(z)-\pi_{n}(z)\right| \leqq \frac{M^{\prime \prime \prime}}{R_{1}^{n}},
$$

where $M^{\prime \prime \prime}$ depends on $r$ but not on $z$. This inequality holds then for every $z$, provided merely that $z$ is interior to $C$ and at a distance at least $r$ from the boundary of $C$. The inequality thus holds, for properly chosen $M^{\prime \prime \prime}$, for $z$ on an arbitrary Jordan curve $\Gamma^{\prime}$ interior to $C$. Since $R_{1}<R$ and $\Gamma^{\prime}$ are both arbitrary, Theorem V follows from Theorem I and the results of Carathéodory previously used.

It will be noticed that the situation is more complicated here than previously, for $C$ is no longer a Jordan region. Nevertheless the results of Carathéodory apply, for $D$ is a region whose complete boundary lies in the (closed) region $C$, and hence when $\Gamma^{\prime}$ is uniformly near the boundary of $C$, the curve $C_{R_{1}^{\prime}}$ is uniformly near the curve $C_{R_{1}}$. The curves $C_{R_{1}}$ and $C_{R_{1}^{\prime}}$ are the transforms of the circle $|w|=R_{1}$ when the region $D$ and the exterior of $\Gamma^{\prime}$ respectively are mapped onto $|w|>1$ so that the points at infinity correspond to each other. The region $D$ is the kernel of the exteriors of a properly chosen

* For the method used here, compare Kellogg, Potential Theory, Berlin, 1929, p. 268. The method is due to Zaremba; see Bouligand, Mémorial des Sciences Mathématiques, Fascicule 11, pp. 20-21. 
sequence of curves $\Gamma^{\prime}$. Carathéodory's Satz $\mathrm{V}$ applies directly, it being noticed that the convergence of the functions involved is uniform.*

For the particular case $p=2, n(z) \equiv 1$, Theorem $\mathrm{V}$ treats the general method of approximation used by Bergmann, $\dagger$ Bochner, $\downarrow$ and Carleman. $\S$ Again we have approximating polynomials of the form

$$
\pi_{n}(z)=a_{0} P_{0}(z)+a_{1} P_{1}(z)+\cdots+a_{n} P_{n}(z),
$$

where the polynomials $P_{i}(z)$ are independent of $f(z)$ and where the coefficients $a_{i}(i \leqq n)$ are independent of $n$. It will be noticed that here too we have a solution of the problem of Faber mentioned above, and that the present result holds for much more general regions than Jordan regions.

Theorem $\mathrm{V}$ requires more of the function $f(z)$ than that it shall be analytic in the closed region $C$, if the boundary of $C$ divides the plane into more than two distinct regions. Whatever $C$ may be, if $f(z)$ has a singularity exterior to the region $D$ used above, uniform approximation of $f(z)$ in $C$ with an arbitrarily small error is impossible; if $f(z)$ has no singularity except in $D$, then $f(z)$ is analytic interior to some $C_{R}$ and the hypothesis of Theorem $\mathrm{V}$ is satisfied. In particular, if $C$ is an arbitrary limited region whose boundary divides the plane into but two distinct regions, a function $f(z)$ analytic in the (closed) region $C$ is analytic within some curve $C_{R}$, and hence the sequence $\left\{\pi_{n}(z)\right\}$ of polynomials of best approximation to $f(z)$ on $C$ converges uniformly in the closed region $C$, in fact converges interior to $C_{R}$, uniformly on any closed point set interior to $C_{R}$. This same remark applies in the case of Theorem IV, if $C$ is simply connected; compare the footnote to Theorem XII.

We do not carry out the analogue of Theorem $\mathrm{V}$ after mapping of $C$ onto the unit circle $\gamma$; this is easy to do, but due to the arbitrariness of the function $n(z)$, would yield little new. A double integral over $C$ is equal to the corresponding double integral over the interior of $\gamma$, with an additional factor in the integrand, the square of the derivative of the mapping function. It may be remarked that the function $n(z)$ need not be continuous, although for simplicity we have made that assumption, but even in Theorems III and IV as well need merely be positive, integrable, and bounded from zero. We do not, however, mean to imply the complete equivalence of Theorem $\mathrm{V}$ and the analogous theorem obtained by studying approximation measured

\footnotetext{
* The theorem for harmonic functions (used below) corresponding to Theorem I is not an exact analogue of Theorem I, but is to be found as Theorem III of a paper by the present writer, Bulletin of the American Mathematical Society, vol. 33 (1927), pp. 591-598.

$\dagger$ Mathematische Annalen, vol. 86 (1922), pp. 238-271.

$\ddagger$ Mathematische Zeitschrift, vol. 14 (1922), pp. 180-207.

$\S$ Arkiv för Matematik, Astronomi, och Fysik, vol. 17 (1922-23).
} 
over the area interior to $\gamma$. The latter can be obtained with ease by the reader, in the special case corresponding to Theorem $\mathrm{V}$ that $C$ is simply connected.

9. Approximation to harmonic functions. We turn now to the analogues of Theorems I-V, for approximation to harmonic functions by harmonic polynomials. The results and methods are in the main so similar to those already used that we need not enter into full detail. The analogue of Theorem $I$ is not difficult to prove, ${ }^{*}$ provided that $C$ is a Jordan region, and leads $\dagger$ directly to the analogue of Theorem II, also for the case that $C$ is a Jordan region, and even if $C$ is an arbitrary limited region. This result, the analogue of Theorem II, naturally persists even though we consider the harmonic Tchebycheff polynomial with the norm function $n(x, y)$.

The analogue of Theorem IV is likewise easy to prove:

Theorem VI. Let the function $u(x, y)$ be harmonic within $C_{R}$, where $C$ is an arbitrary closed limited simply connected region. Then the sequence of harmonic polynomials $\left\{\pi_{n}(x, y)\right\}$ of best approximation to $u(x, y)$ on $C$ in the sense of least weighted pth powers as measured on $\gamma$ converges to $u(x, y)$ throughout the interior of $C_{R}$, uniformly on any closed point set interior to $C_{R}$.

In the proof of Theorem VI it is essential to know, in order to use Poisson's integral, that for such a function as $u(x, y)$ the functional values on $\gamma$ found by conformal transformation coincide almost everywhere with the functional values found by radial approach to $\gamma$ after conformal transformation. This presents no difficulty, since $\lim _{r \rightarrow 1}, r<1 \psi\left(r e^{i \theta}\right)$ exists for almost all values of $\theta$; such a value $\theta^{\prime}$ corresponds to an accessible point of the boundary of $C$, and the radius $\theta=\theta^{\prime}$ of $\gamma$ corresponds to a Jordan arc interior to $C$ abutting in $\psi\left(e^{i \theta^{\prime}}\right)$. The limit of $u(x, y)$ along this arc is naturally the value of $u(x, y)$ at the end point.

In the proof of Theorem VI there enters the derivative of Green's function for the circle $\gamma$ in the place of the function $1 /(t-w)$ of Theorem IV.

This general situation, of considering best approximation to a harmonic function by harmonic polynomials in the sense of least pth powers as measured on $\gamma$, has recently been studied by Julia (loc. cit.), and important general properties derived.

The analogue of Theorem III is not so simple, for the precise analogue of Cauchy's integral is Green's formula

* See Walsh, Bulletin of the American Mathematical Society, vol. 33 (1927), pp. 591-598.

$\dagger$ Walsh, Bulletin of the American Mathematical Society, vol. 35 (1929), pp. 499-544; especially p. 513. The theorem is there stated and proved only for a Jordan region $C$, but the proof can be given in the more general case. See Theorem III of the paper referred to in the preceding footnote. 


$$
u(x, y)-\pi_{n}(x, y)=\frac{1}{2 \pi} \int_{\Gamma}\left[u(x, y)-\pi_{n}(x, y)\right] \frac{\partial G}{\partial n} d s
$$

where $G$ is Green's function for the region, and comparatively little seems to be known about $\partial G / \partial n$ for an arbitrary rectifiable Jordan curve $\Gamma$. We may take an extreme case, however, and assume the curve $\Gamma$ to have continuous curvature. It follows that $\partial G / \partial n$ is continuous on $\Gamma$, and we may state

THEOREM VII. Let the function $u(x, y)$ be harmonic within $C_{R}$, where the point set $C$ is the interior of the Jordan curve $\Gamma$, assumed to have continuous curvature. Then the sequence of harmonic polynomials $\left\{\pi_{n}(x, y)\right\}$ of best approximation to $u(x, y)$ on $C$ in the sense of least weighted pth powers as measured on $\Gamma$ converges to $u(x, y)$ throughout the interior of $C_{R}$, uniformly on any closed point set interior to $C_{R}$.

This particular measure of approximation for $p=2, n(s) \equiv 1$, has been considered by S. Bernstein, ${ }^{*}$ Brillouin, $\dagger$ and Picone, $\ddagger$ and for this restricted case Theorem VII has been proved by Merriman $\S$ by the present methods.

The analogue of Theorem $\mathrm{V}$ presents not the slightest difficulty:

THEOREM VIII. Let $C$ be an arbitrary limited closed region. If the function $u(x, y)$ is harmonic interior to $C_{R}$, then the sequence $\left\{\pi_{n}(x, y)\right\}$ of harmonic polynomials of best approximation to $u(x, y)$ in the sense of least weighted pth powers over the area $C$ converges to $u(x, y)$ interior to $C_{R}$, uniformly on an arbitrary closed point set interior to $C_{R}$.

Here too we have for the case $p=2, n(x, y) \equiv 1$, approximating polynomials $\pi_{n}(x, y)$ of the form

$$
\pi_{n}(x, y)=a_{0} P_{0}(x, y)+a_{1} P_{1}(x, y)+\cdots+a_{n} P_{n}(x, y),
$$

where the polynomials $P_{i}(x, y)$ are independent of $u(x, y)$ and where the coefficients $a_{i}(i \leqq n)$ are independent of $n$. In particular if $C$ is an arbitrary limited region whose boundary divides the plane into but two distinct regions, a function $u(x, y)$ harmonic in (the closed region) $C$ is harmonic within some curve $C_{R}$, and hence the sequence $\left\{\pi_{n}(x, y)\right\}$ of polynomials of best approximation to $u(x, y)$ on $C$ converges uniformly to $u(x, y)$ in the closed region $C$, in fact converges interior to $C_{R}$, uniformly on any closed point set interior to $C_{R}$.

* Paris Comptes Rendus, vol. 148 (1909), pp. 1306-1308.

$\dagger$ Annales de Physique, vol. 6 (1916), pp. 137-223.

‡ Rendiconti dei Lincei, 1922, pp. 357-359. These three references were unintentionally omitted in the writer's report referred to above, on approximation of harmonic functions by harmonic polynomials.

$\S$ On the expansion of harmonic functions in terms of normal orthogonal harmonic polynomials, not yet published. Presented to the American Mathematical Society in April, 1928. 
10. Generalizations. We make a few remarks about the existence and uniqueness of the polynomials of best approximation. The harmonic Tchebycheff polynomial of best approximation with a norm function unity to a function harmonic interior to an arbitrary Jordan curve $\Gamma$, continuous in the corresponding closed region, has been considered independently by Julia, in a paper presented to the Bologna Congress (September 1928) and not yet published, and by the present writer.* It is of course immaterial here whether we study approximation to $u(x, y)$ on $\Gamma$ or in the closed region $C$ bounded by $\Gamma$. The Tchebycheff polynomial always exists in this case, but is not necessarily unique; this same remark holds for an arbitrary limited region $C$ bounded by a point set $\Gamma$, and whether or not the norm function $n(x, y)$ (continuous on $C$ ) is unity.

In any of the situations of Theorems V-VIII, the harmonic polynomial of best approximation always exists, for in every case boundedness for a sequence of approximating polynomials of the measure of approximation in $C$ implies uniform boundedness of the sequence of polynomials in an arbitrary closed region interior to $C$, and the method as given by Julia applies. In these same situations, the harmonic polynomial of best approximation is likewise unique; this also follows from the method given by Julia for the case of Theorem VI, $n(s) \equiv 1$, in the paper to which reference has been made several times. The method and result apply too in the cases of approximation to analytic functions already taken up, where approximation is measured by an integral. The same method and result apply also for approximation to analytic and harmonic functions in the similar cases to be treated below. The uniqueness of the polynomials of best approximation, it may be pointed out, does not enter in any way into Theorems II-VIII.

It will be noticed that the theorems we have stated for harmonic functions, like those for analytic functions, are not the most general that we might have stated. They involve, in fact, not necessarily the sequence of polynomials of best approximation, but any sequence of polynomials which converges, according to some measure of convergence, like a convergent geometric series. Let us for definiteness describe the situation as in Theorems III-V for approximation to an analytic function, although of course the situation is similar for harmonic functions. We can show (from Theorem I) under certain restrictions on the region $C$, that if $f(z)$ is analytic interior to $C_{R}$, there exists a sequence of polynomials of respective degrees $n$ such that the measure of approximation (considered in any one of several ways) is of the order $1 / R_{1}{ }^{n p}$ for an arbitrary $R_{1}<R$. We can show on the other hand

* Bulletin of the American Mathematical Society, vol. 35 (1929), pp. 499-544; §4. 
that any sequence of polynomials of respective degrees $n$ such that the measure of approximation is of order $1 / R_{1}{ }^{n p}$ for an arbitrary $R_{1}<R$, converges to $f(z)$ not merely on $C$, but throughout the interior of $C_{R}$, uniformly on any closed point set interior to $C_{R}$. This conclusion naturally follows, then, for the polynomials of best approximation.

A similar remark applies to the discussion of $\$ \$ 11$ and 12 below.

It will be noticed too that each of Theorems II-V gives a result relative to the degree of the best approximation to the given function. In Theorem II, for instance, suppose $f(z)$ actually to have a singularity on $C_{R}$. If $R_{1}$ is an arbitrary number less than $R$, we surely have for some $M_{1}$

$$
\left|f(z)-\pi_{n}(z)\right| \leqq M_{1} / R_{1}{ }^{n}, \quad z \text { on } C .
$$

Yet there exists no number $R_{2}$ greater than $R$ such that

$$
\left|f(z)-\pi_{n}(z)\right| \leqq M_{2} / R_{2}{ }^{n}, \quad z \text { on } C .
$$

Similar results for the other measures of approximation can obviously be written down, for analytic functions and for harmonic functions.

11. Approximation to less restricted analytic functions. It is worth noticing that the inequalities we have been using in proving the overconvergence of the sequences of polynomials are precisely the natural equalities that might be used in proving the simple convergence of the approximating polynomials when less stringent requirements are put on the functions approximated. In every case corresponding to Theorems III-VIII where we deal with a Jordan region $C$, if the function to be approximated is analytic or harmonic in the interior of the region $C$ and continuous in the closed region, then the sequence of polynomials of best approximation converges to the function interior to $C$, uniformly on any closed point set interior to $C$. Let us prove this fact, for instance, in the situation corresponding to Theorem III.*

THEOREM IX. Let the function $f(z)$ be analytic interior to $C$, continuous in the corresponding closed region, where $C$ is the interior of the rectifiable Jordan curve $\Gamma$. Then the sequence of polynomials $\left\{\pi_{n}(z)\right\}$ of best approximation to $f(z)$ on $C$ in the sense of weighted pth powers as measured on $\Gamma$ converges to $f(z)$ throughout the interior of $C$, uniformly on any closed point set interior to $C$.

We introduce the following notation for the measure of approximation:

\footnotetext{
* For the sequence of Tchebycheff polynomials, we have convergence to the function approximated, uniformly in the closed region $C$. Compare, for more general regions $C$, the end of $\$ 2$ and the beginning of $\$ 12$.
} 


$$
\epsilon_{n}=\int_{\Gamma}\left|f(z)-\pi_{n}(z)\right|^{p} n(s) d s .
$$

The function $f(z)$ can be uniformly approximated by a polynomial as closely as desired in the closed region $C$, so $\lim _{n \rightarrow \infty} \epsilon_{n}=0$. We have, as in (4),

$$
\int\left|f(z)-\pi_{n}(z)\right|^{p} d s \leqq \int_{\Gamma}\left|f(z)-\pi_{n}(z)\right| p \frac{n(s)}{n^{\prime}} d s \leqq \frac{\epsilon_{n}}{n^{\prime}} .
$$

We apply Cauchy's integral formula, as to (4), and find

$$
\left|f(z)-\pi_{n}(z)\right| \leqq N \epsilon_{n},
$$

where $z$ is restricted to lie on an arbitrary closed point set interior to $C$; the number $N$ depends on this point set but not on $n$. Theorem IX follows at once.

We state explicitly the theorems corresponding to Theorems IV-VIII as well. These theorems are for the most part not entirely new; their present interest lies in their proof by the methods already used. Many of these methods are used here for the first time to prove these particular theorems. The theorems are, however, more general than those proved elsewhere, either with respect to measure of approximation, by introducing a weight function and considering an arbitrary value of $p$ instead of $p=2$, or in treating regions more general than those treated hitherto. References to the literature are to be found in connection with the theorems previously given.

TheOREm Xa. Let the function $f(z)$ be analytic interior to $C$, continuous in the corresponding closed region, where $C$ is the interior of an arbitrary Jordan curve $\Gamma$. Then the sequence of polynomials $\left\{\pi_{n}(z)\right\}$ of best approximation to $f(z)$ on $C$ in the sense of least weighted pth powers as measured on the circumference $\gamma$ converges to $f(z)$ interior to $C$, uniformly on any closed point set interior to $C$.

TheOREM X. Let $C$ be an arbitrary limited simply connected region. If the function $f(z)$ is analytic interior to $C$, continuous in the corresponding closed region, and if $f(z)$ can be approximated in the closed region as closely as desired by a polynomial in $z$, then the sequence $\left\{\pi_{n}(z)\right\}$ of polynomials of best approximation to $f(z)$ in the sense of weighted pth powers as measured on the circumference $\gamma$ converges to $f(z)$ interior to $C$, uniformly on any closed point set interior to $C$.

THEOREM XI. Let $C$ be an arbitrary Jordan region. If the function $f(z)$ is analytic interior to $C$, continuous in the corresponding closed region, then the sequence $\left\{\pi_{n}(z)\right\}$ of polynomials of best approximation to $f(z)$ in the sense of weighted pth powers over the area $C$ converges to $f(z)$ interior to $C$, uniformly on any closed point set interior to $C$. 
In Theorems $\mathrm{Xa}$ and $\mathrm{XI}$ the region $C$ is restricted to be a Jordan region, for it is only for such a region that we know that an arbitrary function $f(z)$ analytic in the interior and continuous in the closed region can be approximated as closely as desired by a polynomial in $z$. The formal requirements for approximation exist in Theorem XI, however, if $C$ is an arbitrary limited region, simply connected or not, and in particular if the weight function $n(z)$ is chosen as unity, then for $p=2$ we have a set of polynomials $\left\{P_{n}(z)\right\}$ belonging to $C$. Any function $f(z)$ which can be approximated arbitrarily closely leads in Theorems $\mathrm{X}, \mathrm{Xa}$, and XI to a series

$$
f(z)=a_{0} P_{0}(z)+a_{1} P_{1}(z)+\cdots
$$

where the polynomials $P_{i}(z)$ depend on $C$ but not on $f(z)$, where the coefficients $a_{i}$ depend only on $f(z)$ and where the series converges to the sum $f(z)$ interior to $C$, uniformly on an arbitrary point set interior to $C$. The partial sums of the series are the polynomials of best approximation.

If $C$ is a circle, the polynomials $P_{n}(z)$ are, except for numerical factors, the polynomials $z^{n}$ respectively.

This remark on the special form of the polynomials $\pi_{n}(z)$ applies also in connection with

THEOREM XII. Let $C$ be an arbitrary limited region. If the function $f(z)$ is analytic interior to $C$, continuous in the corresponding closed region, and if $f(z)$ can be approximated in the closed region as closely as desired* by a polynomial in $z$, then the sequence $\left\{\pi_{n}(z)\right\}$ of polynomials of best approximation to $f(z)$ in the sense of weighted pth powers over the area $C$ converges to $f(z)$ interior to $C$, uniformly on any closed point set interior to $C$.

Theorem XII has the advantage over Theorem X of holding for multiply connected regions.

We refrain from writing down the analogues of Theorems XI and XII obtained by conformal mapping, considering approximation in the sense of

* It may be noticed that here and elsewhere in the present paper this measure of approximation may be taken either in the sense of Tchebycheff or in the sense of weighted $p$ th powers as used in the theorem itself. We remark that analyticity of $f(z)$ in the closed region $C$ does not ensure the satisfaction of the condition of the theorem, except in the case that the boundary of $C$ divides the plane into precisely two regions. Compare Walsh, Mathematische Annalen, vol. 96 (1926), pp. 430-436; 437-450, especially p. 433 and p. 441 , footnote. In particular, if $C$ is not simply connected, the function $f(z)$ can have no singularities interior to a Jordan curve $K$ which lies entirely interior to $C$. For the sequence $\left\{\pi_{n}(z)\right\}$ converges uniformly to $f(z)$ on $K$, hence represents a function analytic everywhere interior to $K$ coinciding with $f(z)$ on $K$ and therefore coinciding with $f(z)$ everywhere interior to $K$. 
least weighted $p$ th powers over the area interior to $\gamma$ in case $C$ is simply connected. Compare the remark made relative to Theorem V.

12. Approximation to less restricted harmonic functions. The theorems on harmonic functions are also not difficult to treat.

We consider first the Tchebycheff measure of approximation. Let $C^{\prime}$ be an arbitrary closed limited region and let $C$ denote the totality of closed limited regions into which the boundary of $C^{\prime}$ divides the plane. The boundary points of $C$ (considered as a set of individual regions, not as a point set) are precisely the boundary points of $C^{\prime}$. If the function $u(x, y)$ is harmonic interior to $C^{\prime}$, continuous in the closed region, it is immaterial whether we approximate $u(x, y)$ in $C^{\prime}$, or on the boundary of $C$ or $C^{\prime}$. On all of these point sets, the maximum of

$$
\left|u(x, y)-p_{n}(x, y)\right|
$$

where $p_{n}(x, y)$ is a harmonic polynomial, is one and the same. This fact also holds for the approximation of $u(x, y)$ on $C$, if by $u(x, y)$ we mean not necessarily the harmonic extension of the given function $u(x, y)$ defined in $C^{\prime}$, but the function $u(x, y)$ defined on the entire point set $C$ as the function which is continuous on $C$, which in the boundary points of $C$ (i.e. considered as a set of regions, not as a point set) takes on the values of the given function $u(x, y)$, and which is harmonic in the interior points of $C{ }^{*}$ If the sequence of Tchebycheff harmonic polynomials, or any other set of harmonic polynomials, converges to $u(x, y)$ on the boundary of $C^{\prime}$, that sequence converges on the entire point set $C$ to the function $u(x, y)$ as just defined. Only such a function $u(x, y)$ actually or potentially defined on $C$ can be uniformly approximated in the closed region $C^{\prime}$ by harmonic polynomials. A necessary and sufficient condition that such a function can be so approximated, which is a necessary and sufficient condition for the convergence of the sequence of Tchebycheff polynomials to the function, is contained in the second paper just referred to.

THEOREM XIII. Let the function $u(x, y)$ be harmonic interior to $C$, contimuous in the corresponding closed region, where $C$ is the interior of an arbitrary Jordan curve $\Gamma$ with continuous curvature. Then the sequence of harmonic polynomials $\left\{\pi_{n}(x, y)\right\}$ of best approximation to $u(x, y)$ on $C$ in the sense of weighted pth powers as measured on $\Gamma$, converges to $u(x, y)$ throughout the interior of $C$, uniformly on any closed point set interior to $C$. p. 597 .

* Compare Walsh, Bulletin of the American Mathematical Society, vol. 33 (1927), pp. 591-598; Also Crelle's Journal, vol. 159 (1928), pp. 197-209; pp. 204-205. 
Theorem XIV. Let $C$ be an arbitrary limited simply connected region whose boundary is also the boundary of an infinite region. If the function $u(x, y)$ is harmonic interior to $C$, continuous in the closed region, then the sequence $\left\{\pi_{n}(x, y)\right\}$ of harmonic polynomials of best approximation to $u(x, y)$ on $C$ in the sense of weighted pth powers as measured on the circumference $\gamma$ converges to $u(x, y)$ interior to $C$, uniformly on any closed point set interior to $C .{ }^{*}$

In particular if the weight function is unity and $p=2$, we have a set of harmonic polynomials $\left\{P_{n}(x, y)\right\}$ belonging to the region $C$, and the sequence $\left\{\pi_{n}(x, y)\right\}$ corresponds to the partial sums of the series

$$
u(x, y)=a_{0} P_{0}(x, y)+a_{1} P_{1}(x, y)+\cdots,
$$

where the coefficients $a_{i}$ depend only on $u(x, y)$. This remark can be extended to the most general limited region.

THEOREM XV. Let $C$ be an arbitrary limited simply connected region. If the function $u(x, y)$ is harmonic interior to $C$, continuous in the corresponding closed region, and if $u(x, y)$ can be uniformly approximated in the closed region as closely as desired by a harmonic polynomial, then the sequence $\left\{\pi_{n}(x, y)\right\}$ of harmonic polynomials of best approximation to $u(x, y)$ on $C$ in the sense of weighted pth powers as measured on the circumference $\gamma$ converges to $u(x, y)$ interior to $C$, uniformly on any closed point set interior to $C$.

THEOREM XVI. Let $C$ be an arbitrary limited region whose boundary is also the boundary of an infinite region. If the function $u(x, y)$ is harmonic interior to $C$, continuous in the closed region, then the sequence $\left\{\pi_{n}(x, y)\right\}$ of harmonic polynomials of best approximation to $u(x, y)$ on $C$ in the sense of weighted pth powers as measured over the area $C$ converges to $u(x, y)$ interior to $C$, uniformly on any closed point set interior to $C$.

If $p=2$ and the weight function is unity, we have a set $\left\{P_{n}(x, y)\right\}$ of harmonic polynomials belonging to the region $C$, and the sequence $\left\{\pi_{n}(x, y)\right\}$ corresponds to the partial sums of the series

$$
u(x, y)=a_{0} P_{0}(x, y)+a_{1} P_{1}(x, y)+\cdots,
$$

where the coefficients $a_{i}$ depend only on $u(x, y)$ and where the polynomials $P_{n}(x, y)$ are normal and orthogonal with respect to the area $C$. When $C$ is

* Here we apply a general theorem on approximation to harmonic functions; see the paper by the present writer in Crelle's Journal to which reference has just been made. That paper was presented to the American Mathematical Society in December, 1926, and an abstract of it was published in the Bulletin of that Society, vol. 33 (1927), p. 150. Compare also Julia, a paper presented to the Bologna Congress, September, 1928, and not yet published. 
a circle, the polynomials $P_{n}(x, y)$ are precisely those which lead to the expansion of $u(x, y)$ in Fourier's series. In this case, the expansion of $u(x, y)$ is the same whether we have best approximation in the sense of least squares over the area $C$ or over the circumference of $C$.

TheOREM XVII. Let $C$ be an arbitrary limited region. If the function $u(x, y)$ is harmonic interior to $C$, continuous in the closed region, and if $u(x, y)$ can be approximated in the closed region as closely as desired by a harmonic polynomial, ${ }^{*}$ then the sequence $\left\{\pi_{n}(x, y)\right\}$ of harmonic polynomials of best approximation to $u(x, y)$ on $C$ in the sense of weighted pth powers as measured over the area $C$ converges to $u(x, y)$ interior to $C$, uniformly on any closed point set interior to $C$.

Theorem XVII holds even for harmonic functions in $n$ dimensions. In particular if $C$ is bounded by a simple closed surface, and if $u(x, y, \cdots)$ is harmonic in the closed region, the hypothesis of the theorem is satisfied.

Nowhere in the suggested proofs of Theorems IX-XVII is there used the fact that the sequence $\left\{\pi_{n}(z)\right\}$ or $\left\{\pi_{n}(x, y)\right\}$ is the sequence of polynomials of best approximation, although the existence and uniqueness of those polynomials of best approximation are assured as in connection with the previous theorems. It is sufficient for the conclusion of Theorems IX-XVII in each case if $\epsilon_{n}$, the measure of approximation of the polynomial to the function (compare the proof of Theorem IX), approaches zero with $1 / n$. Moreover, we have assumed for simplicity that the functions $f(z)$ and $u(x, y)$ are continuous in the closed regions considered, but that restriction can be materially lightened in the several cases, as an inspection of the proofs will show.

13. Application to the Dirichlet problem. As Julia points out, the fact that for a Jordan region the harmonic polynomial of best approximation to a harmonic function, either in the sense of Tchebycheff or in the sense of weighted $p$ th powers as measured on $\boldsymbol{\gamma}$ or on $\Gamma$, refers merely to the boundary of the region, gives us a theoretical solution of the Dirichlet problem. The Tchebycheff harmonic polynomials of best approximation are the same for the boundary values and for the harmonic function taking on those boundary values (i.e. the corresponding solution of the Dirichlet problem), and the sequence of

* It will be noticed in particular that if $C$ is not simply connected, the function $u(x, y)$ and its harmonic extension can have no singularities interior to an arbitrary Jordan curve $K$ which lies entirely interior to $C$. For the sequence $\left\{\pi_{n}(x, y)\right\}$ converges uniformly to the limit $u(x, y)$ on $K$, hence represents a function harmonic everywhere interior to $K$, which coincides at some points interior to $K$ (namely at points interior to both $K$ and $C$ ) with $u(x, y)$, and which therefore coincides with $u(x, y)$ everywhere interior to $K$. 
those polynomials or of the other polynomials mentioned converges interior to the given region to the solution of the Dirichlet problem which corresponds to the given boundary values. With the Tchebycheff method of approximation the actual determination of the polynomials of best approximation seems to be extremely difficult. In the cases of Theorems XIII-XV the formulas for the polynomials of best approximation $(p=2, n(s) \equiv 1)$ involve only comparatively simple line integrals, so the method of solving the Dirichlet problem has some practical value. The numerical computation can in certain cases actually be carried out, as has been done notably by Bergmann.*

The question of the actual convergence on $\Gamma$ of the approximating sequences that we have been considering in $\$ \S 11$ and 12 is an interesting one. $\dagger$ If approximation is in the sense of Tchebycheff, the sequence of polynomials of best approximation naturally converges uniformly in the closed region $C$ in every case we have treated. If approximation is measured by a line integral, in every case the measure $\epsilon_{n}$ of approximation approaches zero with $1 / n$, and it follows that we have

$$
\text { q } \lim _{n \rightarrow \infty} \pi_{n}(z)=f(z), \quad \underset{n \rightarrow \infty}{\operatorname{qlim}} \pi_{n}(x, y)=u(x, y),
$$

in the sense of quasiconvergence $\neq$ on $\Gamma$. That is to say, from every subsequence of the $\pi_{n}(z)$ [or $\left.\pi_{n}(x, y)\right]$ can be extracted a new subsequence which converges almost everywhere on $\Gamma$ to $f(z)$ [or $u(x, y)]$.

If $\epsilon_{n}$ is an integral taken over $\Gamma$, measure (in the term quasiconvergence) is to be taken in the sense of arc length on $\Gamma$. If $\epsilon_{n}$ is an integral over $\gamma$, we have quasiconvergence on $\gamma$ and hence on $\Gamma$, but measure is here to be taken in the sense of arc length on $\gamma$. Nevertheless, even in this case, measure may be taken in the sense of arc length on $\Gamma$ if $\Gamma$ is rectifiable.

* See for instance Zeitschrift für Angewandte Mathematik und Mechanik, vol. 8 (1928), pp. 402-413.

$\dagger$ There is also the corresponding question in $\$ \$ 1-10$ of the convergence on $C_{R}$; this question seems to be entirely unanswered in the general case. S. Bernstein has established some results in this connection for approximation on a point set $C$ which is an interval of the axis of reals.

$\ddagger$ See Walsh, these Transactions, vol. 32 (1930), pp. 335-390.

HaRVARD UNIVERSITY, Cambridge, Mass. 